\title{
A follow-up study of children infected with SARS-CoV-2 from western China
}

\begin{abstract}
Hongmei Xu ${ }^{1,2,3 \#}$, Enmei Liu ${ }^{2,3,4 \#}$, Jun Xie ${ }^{2,3,4}$, Rosalind L. Smyth ${ }^{5}$, Qi Zhou ${ }^{6}$, Ruiqiu Zhao ${ }^{1,2,3}$, Na Zang ${ }^{2,3,4}$, Xiaoru Long ${ }^{1,2,3}$, Yuyi Tang ${ }^{2,3,4}$, Janne Estill ${ }^{7}$, Shu Yang ${ }^{8}$, Jing Zhu ${ }^{1,2,3}$, Xiaofeng Yan', Fang Gong ${ }^{10}$, Wenguang Tian ${ }^{10}$, Xiaqia Zhou ${ }^{10}$, Yunbo Mo ${ }^{11}$, Hongzhou Xiao ${ }^{11}$, Zhengzhen Tang ${ }^{12}$, Yanni Chen ${ }^{13}$, Yi Wang $^{13}$, Yuxia Cui ${ }^{14}$, Xiuling Fang ${ }^{15}$, Feiyu Li ${ }^{16}$, Yong Tian ${ }^{17}$, Peibo Li $^{17}$, Quanmin Deng ${ }^{18}$, Chongsong Ren $^{19}$, Ronghui $\mathrm{He}^{19}$, Yi Li ${ }^{20}$, Hong Qin ${ }^{21}$, Aiguo Wang ${ }^{22}$, Hongli Deng ${ }^{23}$, Jianguo Wu ${ }^{24}$, Wenbo Meng ${ }^{25}$, Weiguo $\mathrm{Li}^{2,3,4}$, Yao Zhao ${ }^{2,3,4}$, Zhengxiu Luo ${ }^{2,3,4}$, Zijun Wang ${ }^{26}$, Yaolong Chen ${ }^{26}$, Gary Wing Kin Wong ${ }^{27}$, Qiu $\mathrm{Li}^{2,3,28}$
\end{abstract}

${ }^{1}$ Department of Infection, Children's Hospital of Chongqing Medical University, Chongqing 400014, China; ${ }^{2}$ National Clinical Research Center for Child Health and Disorders, Ministry of Education Key Laboratory of Child Development and Disorders, China International Science and Technology Cooperation Base of Child Development and Critical Disorders, Chongqing 400014, China; ${ }^{3}$ Chongqing Key Laboratory of Pediatrics, Chongqing 400014, China; ${ }^{4}$ Department of Respiratory Medicine, Children's Hospital of Chongqing Medical University, Chongqing 400014, China; ${ }^{5}$ UCL Great Ormond St Institute of Child Health, Great Ormond Street Hospital, London, UK; ${ }^{6}$ The First School of Clinical Medicine, Lanzhou University, Lanzhou 730000, China; ${ }^{7}$ Institute of Global Health, University of Geneva, Geneva, Switzerland; Institute of Mathematical Statistics and Actuarial Science, University of Bern, Bern, Switzerland; ${ }^{8}$ College of Medical Information Engineering, Chengdu University of Traditional Chinese Medicine, Chengdu 611137, China; ${ }^{9}$ Department of Infection, Chongqing Public Health Medical Center, Chongqing 400036, China; ${ }^{10}$ Department of Pediatrics, Yongchuan Hospital of Chongqing Medical University, Chongqing 402460, China; ${ }^{11}$ Department of Pediatrics, Three Gorges Hospital, Chongqing University, Chongqing 404100, China; ${ }^{12}$ Department of Pediatrics, Third Affiliated Hospital of Zunyi Medical University, Zunyi 563003 , China; ${ }^{13}$ Department of Infection, Xi'an Children's Hospital, the Affiliated Children's Hospital of Xi'an Jiaotong University, Shaanxi 710003, China; ${ }^{14}$ Department of Pediatrics, Guizhou Provincial People's Hospital, Guiyang 550002, China; ${ }^{15}$ Department of Pediatrics, Tongchuan Mining Bureau Central Hospital, Tongchuan 727000, China; ${ }^{16}$ Department of Pediatrics, Xi'an Eighth Hospital, Xi'an 710065, China; ${ }^{17}$ Department of Pediatrics, The People's Hospital of Xiushan County, Chongqing 409900, China; ${ }^{18}$ Department of Pediatrics, The People's Hospital of Deyang City, Deyang 618000, China; ${ }^{19}$ Department of Pediatrics, Nanchong Central Hospital, Nanchong 637000, China; ${ }^{20}$ Department of Pediatrics, Suining Central Hospital, Suining 629000, China; ${ }^{21}$ Department of Pediatrics, The People's Hospital of Fengdu County, Chongqing 408200, China; ${ }^{22}$ Department of Pediatrics, The People's Hospital of Kaizhou District, Chongqing 405400, China; ${ }^{23}$ Department of Pediatrics, The People's Hospital of Hechuan District, Chongqing 401520, China; ${ }^{24}$ Department of Pediatrics, The People's Hospital of Fengjie County, Chongqing 404600, China; ${ }^{25}$ Special Minimally Invasive Surgery Department, the First Hospital of Lanzhou University, Lanzhou 730000, China; ${ }^{26}$ Evidence-Based Medicine Center, School of Basic Medical Sciences, Lanzhou University, Lanzhou 730000, China; ${ }^{27}$ Department of Pediatrics, The Chinese University of Hong Kong, Hong Kong, China; ${ }^{28}$ Department of Nephrology, Children's Hospital of Chongqing Medical University, Chongqing 400014, China Contributions: (I) Conception and design: GWK Wong, Y Chen, Q Li; (II) Administrative support: Q Li, H Xu, E Liu; (III) Provision of study materials or patients: J Xie, R Zhao, N Zang, X Long, Y Tang, J Zhu, X Yan, F Gong, W Tian, X Zhou, Y Mo, H Xiao, Z Tang, Y Chen, Y Wang, Y Cui, X Fang, F Li, Y Tian, P Li, Q Deng, C Ren, R He, Y Li, H Qin, A Wang, H Deng, J Wu; (IV) Collection and assembly of data: Q Zhou, J Estill, W Li, Z Luo, Y Zhao; (V) Data analysis and interpretation: RL Smyth, S Yang, W Meng, Z Wang; (VI) Manuscript writing: All authors; (VII) Final approval of manuscript: All authors.

\#These authors contributed equally to this article.

Correspondence to: Yaolong Chen. evidence-based Medicine Center, School of Basic Medical Sciences, Lanzhou University, Lanzhou 730000, China. Email: chenyaolong@lzu.edu.cn; Gary Wing Kin Wong. Department of Pediatrics, The Chinese University of Hong Kong, Hong Kong, China. Email: xingkinwong@cuhk.edu.hk; Qiu Li. National Clinical Research Center for Child Health and Disorders, Children’s Hospital of Chongqing Medical University, Chongqing 400014, China. Email: liqiu_21@126.com.

Background: To clarify the characteristic and the duration of positive nucleic acid in children infected with severe acute respiratory syndrome coronavirus 2 (SARS-CoV-2), including asymptomatic children.

Methods: A total of 32 children confirmed with SARS-CoV-2 infection between January 24 and February 12, 2020 from four provinces in western China were enrolled in this study and followed up until discharge

(c) Annals of Translational Medicine. All rights reserved.

Ann Transl Med 2020;8(10):623 | http://dx.doi.org/10.21037/atm-20-3192 
and quarantine 14 days later.

Results: Eleven children (34\%) were asymptomatic, among whom six children had normal computed tomographic (CT) scan images. Age and gender were not associated with clinical symptoms or the results of CT scan in children infected with SARS-CoV-2. The concentrations of white blood cells and neutrophils were higher in children with asymptomatic infection than in children with clinical symptoms or CT abnormalities. Patients who presented with CT abnormalities had lower D-dimer or lower total bilirubin than those who had normal CT scan but clinical symptoms. All children recovered and no one died or was admitted to the pediatric intensive care unit (PICU). The mean duration of positive SARS-CoV-2 nucleic acid was 15.4 ( $\mathrm{SD}=7.2$ ) days and similar for both asymptomatic children and children with symptoms or CT abnormalities. We found a significant negative correlation between the lymphocyte count and the duration of positive nucleic acid test.

Conclusions: Children with asymptomatic infection should be quarantined for the same duration as symptomatic patients infected with SARS-CoV-2. The clinical significance and mechanism behind the negative correlation between the number of lymphocytes and the duration of positive SARS-CoV-2 needs further study.

Keywords: Coronavirus; children; asymptomatic infection; western China

Submitted Apr 07, 2020. Accepted for publication Apr 30, 2020.

doi: $10.21037 / \mathrm{atm}-20-3192$

View this article at: http://dx.doi.org/10.21037/atm-20-3192

\section{Introduction}

SARS-CoV-2 was first detected when a cluster of patients with pneumonia of unknown cause emerged in Wuhan, China in December, 2019. On February 11, the World Health Organization (WHO) officially named the disease as "Corona Virus Disease hyphen one nine" (COVID-19) (1-6). As the number of infections and deaths continued to rise, WHO declared COVID-19 as a global pandemic on March 11, 2020 (7), and is causing serious health and economic impact in 179 countries and regions worldwide. COVID-19, together with severe acute respiratory syndrome (SARS) and Middle East respiratory syndrome (MERS), is a disease caused by beta-coronavirus. However, the mortality rate of COVID-19 is lower than that of SARS (more than $40 \%$ in people over 60 years old) and MERS $(30 \%)(8-11)$.

At present, many papers have reported on the epidemiology and clinical characteristics of COVID-19. However, most studies have so far focused on adults (12-14) and several case series of children have been limited by small sample size or the lack of follow-up, in particular for asymptomatic patients (15-20). Children, compared with adults, have a developing immune system and different physical characteristics, thus it is not appropriate to directly use evidence from adults to guide clinical practice of
COVID-19 in children. Therefore, this is the first followup study that included 32 children infected with SARS$\mathrm{CoV}-2$ from multiple centers in four provinces of western China, to clarify the clinical characteristics, the duration of positive nucleic acid tests and their correlation with lymphocyte counts between children with asymptomatic infection and those with clinical symptoms, thus providing evidence to guide management of children infected with SARS-CoV-2. And we present the following article in accordance with the STROBE reporting checklist (available at http://dx.doi.org/10.21037/atm-20-3192).

\section{Methods}

\section{Study design and patients}

This multicenter retrospective study was approved by the Ethics Committee of Children's Hospital of Chongqing Medical University (No. 2020-002). Given the urgency in policy and clinical decision-making for COVID-19 and difficulty of confirming information for most patients, informed consent was exempted for all patients after discussion and approval of the above-mentioned committee. We recruited all the children (total 32, aged less than 18 years) with confirmed SARS-CoV-2 infection between January 24 and February 12, 2020 in several local hospitals 


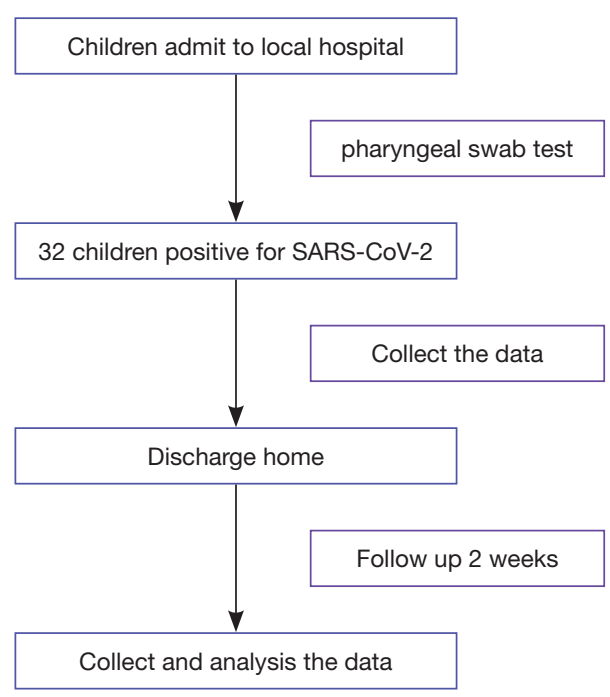

Figure 1 The flowchart of children enrolled in this study.

designated for the diagnosis and treatment of COVID-19 from Chongqing Municipality, Guizhou Province, Shaanxi Province and Sichuan Province. All cases were diagnosed based on the WHO interim guidance (21). And all the cases were followed up two weeks later after discharge in the local hospital (see the Figure 1).

\section{RT-PCR detection}

Specimens of throat swab were obtained and sent to the Chinese Centers for Disease Control and Prevention (CDC) for testing in a unified and standardized way of transportation. RNA was extracted and tested by real-time RT-PCR with SARS-CoV-2 specific primers and probes. The Chinese Center for Disease Control and Prevention recommends: RT-PCR targeting open reading frame $(\mathrm{ORF} 1 \mathrm{ab})$, primers and probes of nucleoprotein $(\mathrm{N})$ gene region to test for SARS-CoV-2. Two genes were targeted during the real-time RT-PCR assay: Target 1 (ORF1ab): forward primer (F) CCCTGTGGGTTTTACACTTAA, reverse primer (R) ACGATTGTGCATCAGCTGA, and fluorescent probe (P) 5'-FAM-CCGTCTGCGGTAT GTGGAAAGGTTATGG-BHQ1-3'; and Target 2 (N): forward primer (F): GGGGAACTTCTCCTGCTAGAAT, reverse primer (R) CAGACATTTTGCTCTCAAGCTG, and fluorescent probe (P) 5'-FAM-TTGCTGCTGCTTG ACAGATT-TAMRA-3'. The results were classified into three categories: a cycle threshold value (Ct-value) less than 37 was defined as a positive test result, and a Ct-value of 40 or more was defined as a negative test. A medium load, defined as a Ct-value of 37 to less than 40 , required confirmation by retesting. If the repeated Ct-value was less than 40 and an obvious peak was observed, or if the repeated Ct-value was less than 37 , the retest was deemed positive (22).

\section{Data collection}

We obtained the electronic medical records of the confirmed COVID-19 children from the Hospital Information System (HIS) and Laboratory Information System (LIS) of each designated hospital for diagnosis and treatment of COVID-19. We extracted the following five types of information: (I) history of exposure: history of traveling and close contacts with areas heavily affected by COVID-19 (Wuhan), history of exposure to the Huanan seafood market, or family cluster of the disease; (II) demographic characteristics: age, gender, vital signs, weight, height, and comorbidities; (III) laboratory findings: routine blood test results, blood biochemistry, coagulation function, infection markers and detection results of common respiratory pathogens; (IV) radiological findings: chest X-ray and CT scans; and (V) clinical outcomes: Recovery was defined as the patient being discharged after having normal body temperature for more than three days, no respiratory symptoms, CT scan changes resolved, and two consecutive negative test results of SARS-CoV-2 (with at least one day between tests). Asymptomatic infection was defined as the patient being tested positive for SARS-CoV-2 without manifesting any clinical symptoms or abnormal chest imaging findings. Severe cases were defined as having at least one of the following: (I) increased respiratory rate, dyspnea, or cyanosis of the lips; (II) pulse oxygen saturation $\leq 93 \%$, or oxygen concentration $\leq 300 \mathrm{mmHg}$ in arterial partial pressure inspiration, or partial pressure of oxygen in arterial $\leq 300 \mathrm{mmHg}$ in inspiration; or (III) lobular lesion or lesion with a progression to $>50 \%$ within 48 hours. If data were missing, we obtained the information by direct communication with attending doctors and other healthcare providers. Missing data were excluded from statistical analyses. All data were extracted by two physicians (Qi Zhou and Weiguo Li) independently, any disagreement was resolved by consensus or consulting a third researcher (Hongmei Xu).

\section{Statistical analysis}

All statistical analyses were performed using SPSS 23.0 
software. Categorical variables were described as absolute numbers and percentages, and continuous variables were described using means and standard deviations. When the data were normally distributed, $t$-test was used to compare the mean of the two samples; otherwise, the rank sum test was used. When comparing multiple groups, we used variance analysis or covariance analysis. $\chi^{2}$ test or Fisher exact probability test were used for categorical data. The results of correlation analysis were presented as a scatter plot. We calculated Pearson correlation coefficients or partial correlation coefficients after controlling the confounding factors such as gender and age. $\mathrm{P}$ values less than 0.05 was considered statistically significant.

\section{Results}

\section{Characteristics of epidemiology and demography}

Clinical data of 32 children with confirmed SARS-CoV-2 infection were collected in this study, with 20 (63\%) patients from Chongqing and 3 (9\%) patients from Shaanxi Province. The average age of the patients was 9 years (SD $=4.7)$, of which $10(31 \%)$ patients were under six years of age, $11(34 \%)$ aged between 6 to 12 years and 11 (34\%) aged between 12 and 18 years. Seventeen (53\%) patients were male. Only 3 patients had underlying comorbidities: 1 had thrombocytopenia, 1 had Down's syndrome and 1 had intestinal atresia. The vital signs on admission including average temperature, respiratory rate and heart rate were $36.7^{\circ} \mathrm{C}(\mathrm{SD}=0.5), 23 \mathrm{bpm}(\mathrm{SD}=4)$ and $95 \mathrm{bpm}(\mathrm{SD}=12)$, respectively. Twelve (38\%) patients had a history of travel or living in the affected area and 29 (91\%) patients with known cases among family members. All patients received antiviral treatment, of which interferon (94\%) and lopinavir/ritonavir tablets (47\%) were the most commonly used (Table 1).

\section{Clinical characteristics and radiological findings}

Of the confirmed 32 patients, 11 children (34\%) patients were asymptomatic with $6(19 \%)$ asymptomatic patients had normal CT scan. At admission, the most frequently experienced symptoms were fever (38\%) and cough (57\%). According to CT scans, 19 (59\%) patients showed abnormalities in the lungs (suggesting pneumonia), of whom 3 (9\%) patients showed bilateral pneumonia, 8 (25\%) patients showed unilateral pneumonia and $8(25 \%)$ patients showed multiple mottling and ground-glass opacity. Five (16\%) patients were found to have only CT abnormalities without clinical symptoms (Table 2).

\section{Laboratory findings}

We analyzed the first laboratory test on admission, leucocytes were above the normal range in $2(6 \%)$ patients and below the normal range in $1(3 \%)$ patient, with an average number of total leucocytes being $6.1 \times 10^{9} / \mathrm{L}(\mathrm{SD}$ $=0.4$ ). The average number of total leucocytes was $8.6 \times 10^{9} / \mathrm{L}$ $(\mathrm{SD}=1.6)$ in children with asymptomatic infection and $5.5 \times 10^{9} / \mathrm{L}(\mathrm{SD}=0.3)$ in children with clinical symptoms and/or CT abnormalities, which is significantly lower than that of asymptomatic patients $(\mathrm{P}=0.011)$. Children with clinical symptoms and/or CT abnormalities were subdivided into three groups as children with CT abnormalities only, clinical symptoms only and both, where no significant difference in leucocyte counts was found. Neutrophils were above the normal range in $8(25 \%)$ patients, with an average value of $3.0 \times 10^{9} / \mathrm{L}(\mathrm{SD}=0.4)$. The average counts of neutrophils was $4.9 \times 10^{9} / \mathrm{L}(\mathrm{SD}=1.6)$ in children with asymptomatic infection and $2.5 \times 10^{9} / \mathrm{L}(\mathrm{SD}=0.2)$ in children with clinical symptoms and/or CT abnormalities, which is significantly lower than that of children with asymptomatic infection $(\mathrm{P}=0.006)$. There were no significant differences between children with $\mathrm{CT}$ abnormalities only, children with clinical symptoms only and both in neutrophils counts. Lymphocytes were below the normal range only in $2(6 \%)$ patients, while $9(28 \%)$ patients showed high lymphocytes counts above the normal range, with an average of $2.6 \times 10^{9} / \mathrm{L}$ $(\mathrm{SD}=0.2)$. No significant differences were found between asymptomatic group and children with symptoms and/ or CT abnormalities. Platelet counts and hemoglobin concentration were basically within the normal range (Table 3).

Most patients had normal liver function: only 1 (3\%) patient had elevated aspartate aminotransferase (AST) and $6(19 \%)$ patients had elevated alanine aminotransferase (ALT), with the maximum value of ALT to be $80 \mathrm{U} / \mathrm{L}$. There were no significant differences between children with asymptomatic infection and those with clinical symptoms and/or CT abnormalities. Albumin reduction was seen in only one patient. Eleven (37\%) patients had increased LDH values. The bilirubin level in children who had CT abnormalities with or without clinical symptoms was lower than those with clinical symptoms only $(\mathrm{P}=0.022)$, with $4(12 \%)$ cases with bilirubin levels above the normal range. For the coagulation function, APTT and PT were within the normal range, and $\mathrm{D}$-dimer was above the normal range in $6(24 \%)$ patients among 25 patients tested, in which the children who had CT abnormalities with or without clinical symptoms showed lower D-dimer than those with clinical 
Table 1 Baseline characteristics and exposure history of children infected with SARS-CoV-2

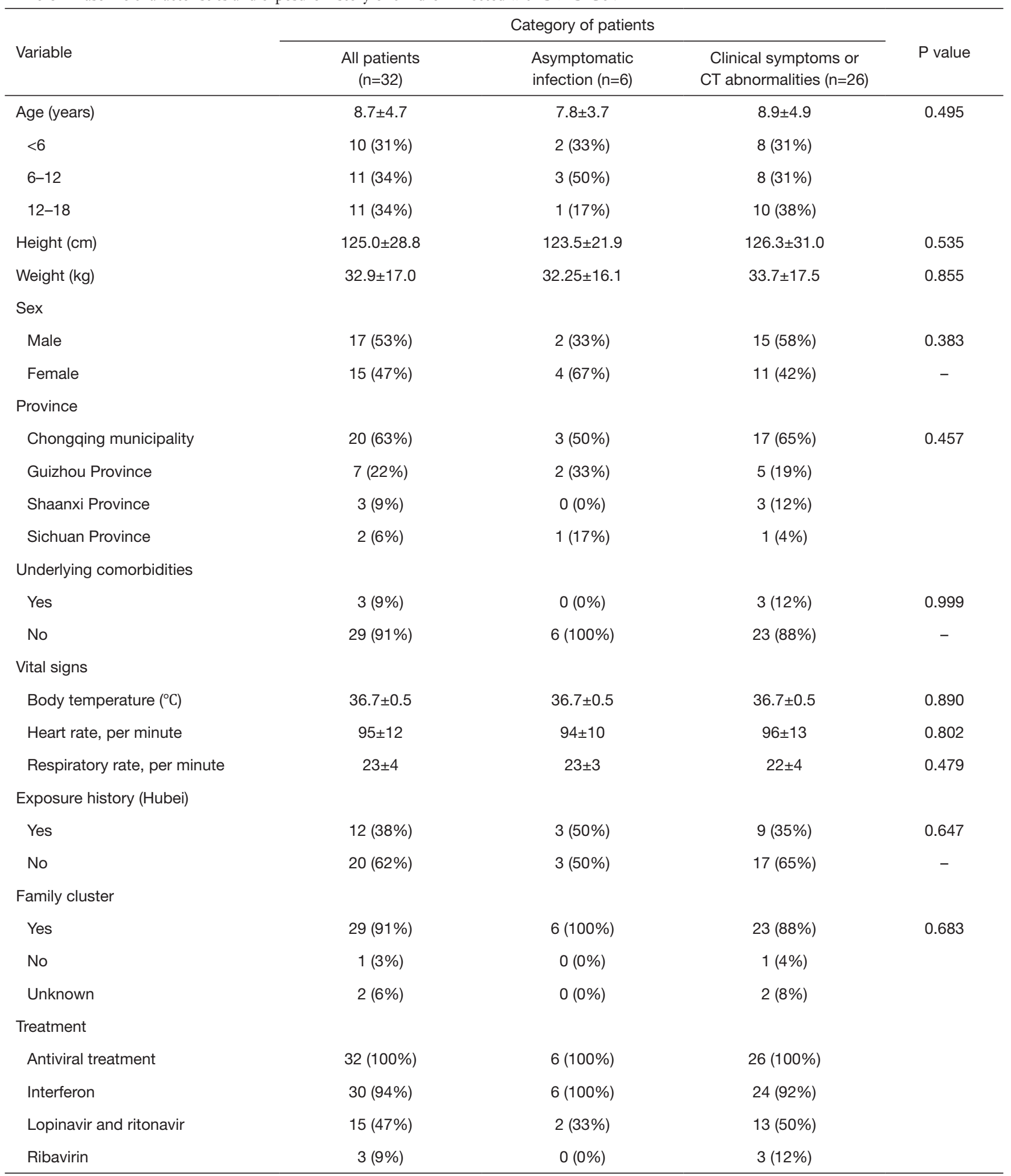

Table 1 (continued) 
Table 1 (continued)

\begin{tabular}{lccc}
\hline & \multicolumn{3}{c}{ Category of patients } \\
\cline { 2 - 4 } Variable & $\begin{array}{c}\text { All patients } \\
(\mathrm{n}=32)\end{array}$ & $\begin{array}{c}\text { Asymptomatic } \\
\text { infection }(\mathrm{n}=6)\end{array}$ & $\begin{array}{c}\text { Clinical symptoms or } \\
\text { CT abnormalities }(\mathrm{n}=26)\end{array}$ \\
\hline Antibiotic treatment & $4(13 \%)$ & $0(0 \%)$ & $4(13 \%)$ \\
Erythromycin & $1(3 \%)$ & $0(0 \%)$ & $1(3 \%)$ \\
Moxifloxacin & $1(3 \%)$ & $0(0 \%)$ & $1(3 \%)$ \\
Ceftriaxone & $1(3 \%)$ & $0(0 \%)$ & $1(3 \%)$ \\
Cefixime & $1(3 \%)$ & $0(0 \%)$ & $0(0 \%)$ \\
Glucocorticoid & $0(0 \%)$ & $0 \%)$ \\
\hline
\end{tabular}

Table 2 Clinical characteristics of children infected with SARS$\mathrm{CoV}-2$ ( $\mathrm{n}=32)$

\begin{tabular}{lc}
\hline Clinical characteristics and CT findings & Number [\%] \\
\hline Clinical characteristics & $8 / 21[38]$ \\
Fever & $12 / 21[57]$ \\
Cough & $6 / 21[29]$ \\
Fever and cough & $3 / 21[14]$ \\
Stuffy nose & $5 / 21[24]$ \\
Runny nose & $3 / 21[14]$ \\
Sore throat & $2 / 21[10]$ \\
Headache & $0 / 21[0]$ \\
Shortness of breath & $1 / 21[5]$ \\
Shiver & $0 / 21[0]$ \\
Choking sensation in chest & $2 / 21[10]$ \\
Fatigue & $11 / 32[34]$ \\
Asymptomatic & $6 / 32[19]$ \\
Asymptomatic and normal CT & $5 / 32[16]$ \\
Only abnormalities on CT & $11 / 21[52]$ \\
More than one symptom & $8[25]$ \\
CT findings & \\
Abnormal & \\
Unilateral pneumonia & {$[59]$} \\
Bilateral pneumonia & 8 [0] \\
\hline
\end{tabular}

symptoms only $(\mathrm{P}=0.031)$ (Table 4). As for bacterial coinfection, procalcitonin was below $0.5 \mu \mathrm{g} / \mathrm{L}$ for all patients who tested and $3(12 \%)$ patients showed increased CRP values among the 26 patients tested (Table 3).

\section{Analysis of duration of positive nucleic acid and recurrence of positive results}

\section{SARS-CoV-2 duration of positive nucleic acid}

By the end of February 29, all 32 patients had been discharged and no severe cases or deaths occurred. Among the 32 children except one lost some retested data who had tested positive for SARS-CoV-2 nucleic acid, by 14 days $52 \%$ (16/31) had a negative nucleic acid test. This was 33\% (2/6) and 56\% (14/25) for asymptomatic patients and symptomatic patients, respectively, which was not statistically different. The mean time from the first positive or onset of symptoms until the first negative nucleic acid test was 15.4 (SD $=7.2$ ) days in 31 patients. No significant difference was identified between children with asymptomatic infection (15.0 days, $\mathrm{SD}=6.7$ ) and children with clinical symptoms (15.5 days, $\mathrm{SD}=7.4)$. In addition, 14 parents, of the enrolled children, who were themselves infected showed a mean duration of 22.7 ( $\mathrm{SD}=10.0)$ days until first negative nucleic acid test, slightly longer than that in children $(\mathrm{P}=0.07)$ (Table 5). The mean duration of until the first negative nucleic acid test was $12.4,18.6$ and 14.9 days in patients under 6,6 to 12 and 12 to 18 years, respectively, with no statistically significant difference between these three age-groups (Table 6). 
Table 3 Laboratory results of children with SARS-CoV-2 infection

\begin{tabular}{|c|c|c|c|c|c|c|c|c|c|}
\hline \multirow[b]{2}{*}{ Characteristic } & \multirow{2}{*}{$\begin{array}{l}\text { Normal } \\
\text { range }\end{array}$} & \multicolumn{6}{|c|}{ Category of patients } & \multicolumn{2}{|c|}{$P$ value } \\
\hline & & $\begin{array}{l}\text { All patients } \\
\quad(\mathrm{n}=32)\end{array}$ & $\begin{array}{l}\text { Asymptomatic } \\
\text { infection }(n=6)\end{array}$ & $\begin{array}{l}\text { Total } \\
(n=26)\end{array}$ & $\begin{array}{l}\text { Only clinical } \\
\text { symptoms } \\
\quad(n=7)\end{array}$ & $\begin{array}{c}\text { Only CT } \\
\text { abnormalities } \\
(n=5)\end{array}$ & $\begin{array}{l}\text { Both } \\
(n=14)\end{array}$ & a & $\mathrm{b}$ \\
\hline \multicolumn{10}{|l|}{ Age and sex } \\
\hline $\operatorname{Sex}(M / F)$ & - & $17 / 15$ & $2 / 4$ & $15 / 11$ & $4 / 3$ & $5 / 0$ & $6 / 8$ & 0.341 & 0.094 \\
\hline \multicolumn{10}{|l|}{ Routine blood tests } \\
\hline Leucocytes $\left(\times 10^{9} / L\right)$ & $3.5-9.5$ & $6.1 \pm 2.3$ & $8.6 \pm 4.0$ & $5.5 \pm 1.1$ & $5.6 \pm 1.4$ & $5.0 \pm 0.7$ & $5.6 \pm 1.1$ & 0.011 & 0.620 \\
\hline Haemoglobin (g/L) & - & $132 \pm 11$ & $131 \pm 4$ & $132 \pm 12$ & $130 \pm 12$ & $142 \pm 8$ & $130 \pm 13$ & 0.753 & 0.236 \\
\hline \multicolumn{10}{|l|}{ Blood biochemistry } \\
\hline ALT (U/L) & $0-45$ & $25 \pm 19$ & $28 \pm 16$ & $25 \pm 20$ & $22 \pm 12$ & $29 \pm 21$ & $25 \pm 23$ & 0.693 & 0.848 \\
\hline AST (U/L) & $0-45$ & $33 \pm 17$ & $34 \pm 8$ & $33 \pm 19$ & $30 \pm 10$ & $34 \pm 6$ & $34 \pm 25$ & 0.896 & 0.871 \\
\hline Albumin (g/L) & $40-55$ & $45.3 \pm 3.1$ & $47.5 \pm 3.2$ & $44.8 \pm 2.9$ & $45.6 \pm 2.8$ & $45.7 \pm 2.2$ & $44.0 \pm 3.1$ & 0.071 & 0.379 \\
\hline $\mathrm{LDH}(\mathrm{U} / \mathrm{L})$ & $120-250$ & $289 \pm 157$ & $313 \pm 116$ & $284 \pm 166$ & $380 \pm 247$ & $213 \pm 7$ & $257 \pm 124$ & 0.709 & 0.180 \\
\hline TB $(\mu \mathrm{mol} / \mathrm{L})$ & $1.7-21$ & $11.5 \pm 9.6$ & $7.3 \pm 2.7$ & $12.4 \pm 10.3$ & $20 \pm 13.6$ & $7.8 \pm 2.4$ & $10.3 \pm 8.6$ & 0.243 & 0.067 \\
\hline
\end{tabular}

Values are presented as mean \pm standard deviation unless otherwise indicated. a: children with asymptomatic infection compared with children with clinical symptoms or CT abnormalities; b: comparing children with clinical symptoms only, CT abnormalities only, and both; or children with clinical symptoms only compared with children with CT abnormalities. *, PCT was available for 23 patients (among which 5 patients with asymptomatic infection).

Table 4 Blood biochemistry results of children with clinical symptoms or/and CT abnormalities

\begin{tabular}{|c|c|c|c|}
\hline $\begin{array}{l}\text { Blood } \\
\text { biochemistry }\end{array}$ & $\begin{array}{c}\text { Only clinical } \\
\text { symptoms }(n=7)\end{array}$ & $\begin{array}{c}\text { With CT } \\
\text { abnormalities } \\
\quad(n=19)\end{array}$ & $P$ value \\
\hline LDH (U/L) & $380 \pm 247$ & $247 \pm 109$ & 0.071 \\
\hline TB $(\mu \mathrm{mol} / \mathrm{L})$ & $20.0 \pm 13.6$ & $9.7 \pm 7.5$ & 0.022 \\
\hline D-dimer $(\mu \mathrm{g} / \mathrm{mL})^{\#}$ & $0.65 \pm 0.60$ & $0.27 \pm 0.18$ & 0.031 \\
\hline
\end{tabular}

Recurrence of positive SARS-CoV-2 nucleic acid results Recurrence of positive SARS-CoV-2 nucleic acid test results were found in 4 (29\%) children 5 to 30 days after the first negative nucleic acid test among 14 children who had subsequent testing after a negative result, of whom one patient was from the asymptomatic group and another three were from children with clinical symptoms and/or CT abnormalities. No significant difference between these two groups was identified (Table 5). And anal swab was tested on 6 children on follow up after discharge and quarantine 14 days, of which 5 tested positive (Table 7). 
Table 5 Time until SARS-Cov-2 nucleic acid turning negative in the children and their parents

\begin{tabular}{|c|c|c|c|c|}
\hline Variable & \multicolumn{3}{|c|}{ Category of patients } & $P$ value \\
\hline Yes & $16(50 \%)$ & $2(33 \%)$ & $14(54 \%)$ & 0.394 \\
\hline \multicolumn{5}{|c|}{ Days of nucleic acid turning negative (d) } \\
\hline Time (d) & $15.4 \pm 7.2$ & $15.0 \pm 6.7$ & $15.5 \pm 7.4$ & 0.853 \\
\hline Children & & $17.6 \pm 7.9$ & & 0.070 \\
\hline
\end{tabular}

*, seventeen parents of the participating children who were infected themselves.

Table 6 Time of SARS-Cov-2 nucleic acid turning negative according to age

\begin{tabular}{lcc}
\hline Age (year) & Time of nucleic acid turning negative $(d)$ & $P$ value \\
\hline$<6$ & $11.5 \pm 3.7$ & 0.360 \\
$6-12$ & $18.6 \pm 9.4$ & \\
$12-18$ & $14.9 \pm 5.9$ & \\
\hline
\end{tabular}

\section{Correlation of the duration until first negative nucleic acid test with blood cells counts}

Among the 32 cases, one patient failed to obtain a complete nucleic acid test result. Another patient showed significantly increased leucocytes after reexamination during hospitalization, which increased from $6.29 \times 10^{9} / \mathrm{L}$ to $14.34 \times 10^{9} / \mathrm{L}$, indicating the presence of co-infection that affected the real situation of routine blood tests, thus these two patients were excluded from data analysis. We found a significant negative correlation between the lymphocyte counts and the time until the first negative nucleic acid, after adjusting for age, gender and length of stay (LOS). In other words, the more lymphocytes, the shorter the time of positive viral nucleic acid, which indirectly reflects the faster virus clearance (Table 8).

\section{Discussion}

The follow-up study is the first time to clarify the clinical characteristics, the time of nucleic acid turning negative and its correlation with lymphocyte counts between children with asymptomatic SARS-CoV-2 infection and those with clinical symptoms.

About $38 \%$ of the children participating in our study had fever and $57 \%$ had cough at the time of admission to hospital. For most children, cough was the first symptom. In CT imaging, pulmonary changes in children were less severe than those in adults, with lower incidence of further progress to severe pneumonia.

Evidence on COVID-19 from adults suggested that at the time of admission, the leucocyte counts was in normal range or decreased in $70 \%$ to $76 \%$ of patients, while the absolute value of lymphocytes was significantly below the normal range in $35 \%$ to $63 \%$ of patients $(12,13)$. In contrast, the results of our study showed that the absolute value of lymphocytes in children with SARS-CoV-2 was mostly normal with only $6 \%$ below the normal range. However, the published expert consensus of COVID-19 for children recommends that clinicians should take decreased lymphocyte counts as one of the diagnostic criteria for suspected cases $(23,24)$. Based on the analysis of these 32 patients in our study, we suggested that using decreased lymphocyte counts for the diagnosis of COVID-19 in children may lead to missed diagnoses of suspected cases and cannot be recommended for diagnosis for children. In addition, our study also found that the total number of white blood cells and absolute neutrophils in children with clinical symptoms and/or CT abnormalities were lower than those with asymptomatic infection, while the lymphocyte counts showed no reduction. Although the platelets showed no difference as the limited cases, it showed the same trend 
Table 7 Recurrence of positive SARS-CoV-2 nucleic acid in children

\begin{tabular}{l} 
Specimens \\
\cline { 2 - 3 } $\begin{array}{c}\text { All patients } \\
(\mathrm{n}=14)\end{array}$
\end{tabular}

as neutrophils. These data suggested that children with SARS-CoV-2 infection may have milder inflammatory response considering that neutrophils were closely related to inflammation, which may be one of the causes of mild illness in children.

Studies from adults showed $98 \%$ of patients had decreased albumin. Liver function in children is less affected by COVID-19 compared with adults (12). Increased D-dimer was found in $24 \%$ of patients who were all symptomatic while asymptomatic patients showed no abnormalities. The level of D-dimer and total bilirubin in children with only clinical symptoms was higher than those who had CT abnormalities with or without clinical symptoms, it seems those with clinical symptoms showed higher D-dimer and total bilirubin, although it showed no difference as only 32 cases were analyzed in this study. These results indicating that D-dimer was kept lower in children with pulmonary imaging abnormalities and that the virus may partially affect the synthesis of bilirubin and D-dimer, of which the significance and mechanism need to be further studied.

As for treatment measures, all included children received antiviral treatment (mostly interferon and lopinavir/ ritonavir) and antibiotics, antifungal agents, glucocorticoid therapy were not used. Previous studies showed that the majority of adults with COVID-19 were given antiviral treatment $(76 \%)$ and antibiotic treatment $(71 \%)$, and some patients needed antifungal agents (15\%) for co-infection control. In some severe cases, oxygen therapy, IVIG and glucocorticoid was need as adjuvant treatment in $76 \%, 27 \%$ and $19 \%$ of the patients, respectively (12).

Previous studies have shown that $26 \%$ to $32 \%$ of patients in adults were admitted to the intensive care unit (ICU) because of their higher oxygen requirement due to hypoxemia. Nosocomial infection was presented in $41 \%$ of adults with high risk of death (4.3\% to $15 \%)(12-14)$. However, in our study, all patients recovered after treatment and no one died or was admitted to the pediatric intensive care unit (ICU) for any other oxygen support. The clinical outcome of COVID-19 in children seems to be significantly better than that in adults.

The mean duration of a positive nucleic acid test, until the first negative result, in children was 15 days, with no significant difference between asymptomatic infected children and children with symptoms or CT abnormalities. Neither was there any statistical difference between that of children and their parents, which was consistent with recent study (25), but as limited cases, more study are needed to confirm the difference between that of children and their parents. These results underline that virus replication occurs for a similar period in children with asymptomatic infection, which implies that children 
with asymptomatic infection should be quarantined for the same duration as symptomatic patients infected with SARS-CoV-2.

No significant difference in the duration of a positive nucleic acid test, until the first negative result was identified between children of different ages, but it seemed that the nucleic acid test became negative faster in children under six years of age, which may be associated with faster virus clearance. However, further research is needed to address this. Recurrence of positive SARS-CoV-2 nucleic acid test results were found in four children 5 to 30 days after the first negative nucleic acid test, all of them however showed improved clinical symptoms, as with adults (26). Recurrence of positive SARS-CoV-2 nucleic acid test results is not necessarily related to recurrent infection and also needs further investigation. In addition, we found a significant negative correlation between the lymphocyte count and the time until the first negative nucleic acid. This result implies that lymphocytes have an impact on the inhibition of SARS$\mathrm{CoV}-2$ replication, which may partially explain the reason why most children infected with SARS-CoV-2 present with only mild illness.

\section{Conclusions}

The clinical characteristics, radiological and laboratory findings, and clinical outcomes of children infected with SARS-CoV-2 are essentially different from the situation in adults. Besides, there were also differences in laboratory findings between patients with asymptomatic infection and patients with symptoms or CT abnormalities. The clinical significance and mechanism behind the negative correlation between the number of lymphocytes and the time of SARS$\mathrm{CoV}-2$ nucleic acid turning negative need to be further studied. This study has limitations including the small sample size and partially missing of data in laboratory test, however, our study can be considered the best evidence yet for management of children infected with SARS$\mathrm{CoV}-2$ considering the current situation of public health emergency. Therefore, prospective and large-sample studies are needed for further research on children infected with SARS-CoV-2 to provide higher-quality and more credible evidence for clinical practice.

\section{Acknowledgments}

We are very grateful to all the medical, nursing and supportive staff of the Chongqing Public Health Medical
Center, Yongchuan Hospital of Chongqing Medical University, Three Gorges Hospital, Third Affiliated Hospital of Zunyi Medical University, Xi'an Children's Hospital, Tongchuan Mining Bureau Central Hospital, the People's Hospital of Xiush'an County, the People's Hospital of Deyang City, Nanchong Central Hospital, Suining Central Hospital, the People's Hospital of Fengdu County, the People's Hospital of Kaizhou District, the People's Hospital of Hechuan District, the People's Hospital of Fengjie County, Guizhou Provincial People's Hospital, for their dedication in looking after the patients.

Funding: National Clinical Research Center for Child Health and Disorders (Children's Hospital of Chongqing Medical University, Chongqing, China) (NCRCCHD2020-EP-01); Special Fund for Key Research and Development Projects in Gansu Province in 2020; The fourth batch of "Special Project of Science and Technology for Emergency Response to COVID-19" of Chongqing Science and Technology Bureau. Special funding for prevention and control of emergency of COVID-19 from Key Laboratory of Evidence Based Medicine and Knowledge Translation of Gansu Province (No. GSEBMKT-2020YJ01); Newton international fellowship from The Academy of Medical Science (NIF004/1012); UK National Institute of Health Research GOSH Biomedical Research Centre; The Fundamental Research Funds for the Central Universities (lzujbky-2020-sp14).

\section{Footnote}

Reporting Checklist: The authors have completed the STROBE reporting checklist. Available at http://dx.doi. org/10.21037/atm-20-3192

Data Sharing Statement: available at http://dx.doi. org/10.21037/atm-20-3192

Conflicts of Interest: All authors have completed the ICMJE uniform disclosure form (available at http://dx.doi. org/10.21037/atm-20-3192). The authors have no conflicts of interest to declare.

Ethical Statement: The authors are accountable for all aspects of the work in ensuring that questions related to the accuracy or integrity of any part of the work are appropriately investigated and resolved. This study strictly complied with the ethical requirements of biomedical research issued by the relevant international and national 
organizations and was approved by the Ethics Committee of Children's Hospital of Chongqing Medical University (No. 2020-002). Given the urgency in policy and clinical decision-making for COVID-19 and difficulty of confirming information for most patients, informed consent was exempted for all patients after discussion and approval of the above-mentioned committee.

Open Access Statement: This is an Open Access article distributed in accordance with the Creative Commons Attribution-NonCommercial-NoDerivs 4.0 International License (CC BY-NC-ND 4.0), which permits the noncommercial replication and distribution of the article with the strict proviso that no changes or edits are made and the original work is properly cited (including links to both the formal publication through the relevant DOI and the license). See: https://creativecommons.org/licenses/by-nc-nd/4.0/.

\section{References}

1. Zhu N, Zhang D, Wang W, et al. A novel coronavirus from patients with pneumonia in China 2019. N Engl J Med 2020;382:727-33.

2. Paules CI, Marston HD, Fauci AS. Coronavirus Infections-More Than Just the Common Cold. JAMA 2020. [Epub ahead of print].

3. Huang C, Wang Y, Li X, et al. Clinical features of patients infected with 2019 novel coronavirus in Wuhan, China. Lancet 2020;395:497-506.

4. Phan LT, Nguyen TV, Luong QC, et al. Importation and human-to-human transmission of a novel coronavirus in Vietnam. N Engl J Med 2020;382:872-4.

5. Li Q, Guan X, Wu P, et al. Early Transmission Dynamics in Wuhan, China, of Novel Coronavirus-Infected Pneumonia. N Engl J Med 2020;382:1199-207.

6. WHO Director-General's remarks at the media briefing on 2019-nCoV on 11 February 2020 2020. Available online: https://www.who.int/dg/speeches/detail/who-directorgeneral-s-remarks-at-the-media-briefing-on-2019-ncovon-11-february-2020

7. Mahase E. Covid-19: WHO declares pandemic because of "alarming levels" of spread, severity, and inaction. BMJ 2020,368:m1036.

8. Prevention and treatment of pneumonia caused by new coronavirus infection. National Health Commission of the People's Republic of China, 2020. Available online: http:// www.nhc.gov.cn/

9. Wu JT, Leung K, Leung GM. Nowcasting and forecasting the potential domestic and international spread of the 2019-nCoV outbreak originating in Wuhan, China: a modelling study. Lancet 2020;395:689-97.

10. Chan-Yeung M, Xu RH. SARS: epidemiology. Respirology 2003;8 Suppl:S9-S14.

11. Ahmed AE. The predictors of 3- and 30-day mortality in 660 MERS-CoV patients. BMC Infect Dis 2017;17:615.

12. Phelan AL, Katz R, Gostin LO. The Novel Coronavirus Originating in Wuhan, China: Challenges for Global Health Governance. JAMA 2020. doi: 10.1001/ jama.2020.1097.

13. Chen N, Zhou M, Dong X, et al. Epidemiological and clinical characteristics of 99 cases of 2019 novel coronavirus pneumonia in Wuhan, China: a descriptive study. Lancet 2020;395:507-13.

14. $\mathrm{Xu} \mathrm{Z}$, Shi L, Wang Y, et al. Pathological findings of COVID-19 associated with acute respiratory distress syndrome. Lancet Respir Med 2020;8:420-2.

15. Lu X, Zhang L, Du H, et al. SARS-CoV-2 Infection in Children. N Engl J Med 2020;382:1663-5.

16. Liu W, Zhang Q, Chen J, et al. Detection of Covid-19 in Children in Early January 2020 in Wuhan, China. N Engl J Med 2020;382:1370-1.

17. Cai J, Xu J, Lin D, et al. A Case Series of children with 2019 novel coronavirus infection: clinical and epidemiological features. Clin Infect Dis 2020. [Epub ahead of print].

18. Wei M, Yuan J, Liu Y, et al. Novel Coronavirus Infection in Hospitalized Infants Under 1 Year of Age in China. JAMA 2020. [Epub ahead of print].

19. Dong Y, Mo X, Hu Y, et al. Epidemiological Characteristics of 2143 Pediatric Patients With 2019 Coronavirus Disease in China. Pediatrics 2020. [Epub ahead of print].

20. Chang D, Lin M, Wei L, et al. Epidemiologic and Clinical Characteristics of Novel Coronavirus Infections Involving 13 Patients Outside Wuhan, China. JAMA 2020. [Epub ahead of print].

21. Corman V, Bleicker T, Brünink S, et al. Diagnostic detection of Wuhan coronavirus 2019 by real-time RTPCR. Geneva: World Health Organization. January 13, 2020. Available online: https://www.who.int/docs/defaultsource/coronaviruse/wuhan-virus-assay-v1991527e512234 1d99287a1b17c111902.pdf. opens in new tab

22. Specific primers and probes for detection 2019 novel coronavirus. Chinese Center for Disease Control and Prevention 2020. Available online: http://ivdc.chinacdc.cn/ kyjz/202001/t20200121_211337.html 


\section{Page 12 of 12}

23. Diagnosis and treatment of pneumonia caused by novel coronavirus (trial version 4). National Health Commission of People's Republic of China 2020. Available online: https://www.nhc.gov.cn/xcs/zhengcwj/202001/4294563 ed35b43209b31739bd0785e67/files/7a9309111267475 a99d4306962c8bf78.pdf

24. Shen K, Yang Y, Wang T, et al. Diagnosis, treatment, and prevention of 2019 novel coronavirus infection in children:

Cite this article as: Xu H, Liu E, Xie J, Smyth RL, Zhou Q, Zhao R, Zang N, Long X, Tang Y, Estill J, Yang S, Zhu J, Yan X, Gong F, Tian W, Zhou X, Mo Y, Xiao H, Tang Z, Chen Y, Wang Y, Cui Y, Fang X, Li F, Tian Y, Li P, Deng Q, Ren C, He R, Li Y, Qin H, Wang A, Deng H, Wu J, Meng W, Li W, Zhao Y, Luo Z, Wang Z, Chen Y, Wong GWK, Li Q. A followup study of children infected with SARS-CoV-2 from western China. Ann Transl Med 2020;8(10):623. doi: 10.21037/atm20-3192

\section{Xu et al. Children with SARS-CoV-2 in western China}

experts' consensus statement. World J Pediatr 2020;7:1-9.

25. Zou L, Ruan F, Huang M, et al. SARS-CoV-2 Viral Load in Upper Respiratory Specimens of Infected Patients. N Engl J Med 2020;382:1177-9.

26. Lan L, Xu D, Ye G, et al. Positive RT-PCR Test Results in Patients Recovered From COVID-19. JAMA 2020. [Epub ahead of print]. 